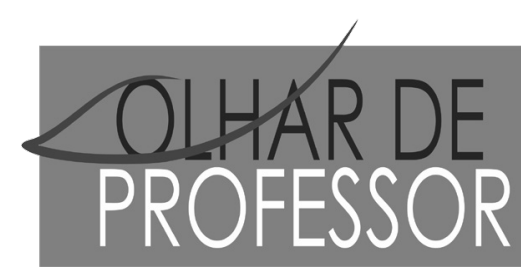

DOI: 10.5212/OLHARPROFR.v.2112.0013

\title{
A INTEGRAÇÃo dAS TDIC NA FORMAÇ̃̃ INICIAL DE PROFESSORES DE MATEMÁTICA NO BRASIL: UMA ANÁLISE A PARTIR DOS PROJETOS PEDAGÓGICOS
}

\author{
INTEGRATION OF ICDT'S IN THE INITIAL EDUCATION OF MATH TEACHERS IN BRAZIL: AN ANALYSIS FROM \\ PEDAGOGICAL PROJECTS
}

\section{LA INTEGRACIÓN DE LAS TDIC EN LA FORMACIÓN INICIAL DE PROFESORES DE MATEMÁTICA EN BRASIL: UN ANÁLISIS A PARTIR DE LOS PROYECTOS PEDAGÓGICOS}

\author{
MARCELI BEHM GOULART ${ }^{\star}$ \\ PRISCILA KABBAZ ALVES DA COSTA* \\ ANA LÚCIA PEREIRA ${ }^{\text {*** }}$
}

\begin{abstract}
Resumo: O artigo é fruto de um projeto de pesquisa que teve como objetivo identificar a formação que os futuros professores de Matemática têm recebido a respeito do uso das tecnologias nos cursos presenciais das universidades públicas brasileira. A partir disso, no presente artigo, temos como objetivo compreender como os cursos de formação inicial de professores de matemática no Brasil abordam a programação de computadores nos projetos pedagógicos. A pesquisa é de natureza qualitativa do tipo análise documental, realizada a partir da análise de projetos pedagógicos dos cursos de Licenciatura em Matemática vigentes no ano de 2016 e 2017. A análise da dados se pautou na análise de conteúdo, do qual emergiram oito categorias. Entretanto, no presente artigo, apresentamos a categoria programação de computadores e suas subcategorias. Como resultados ficou claro que nos PP há uma preocupação de ligar as TDIC com o conteúdo matemático a ser ensinado. Apesar disso não há uma preocupação em fazer a ligação da programação com o pensamento computacional, que deve ser estimulado nos alunos. A formação inicial de professores de matemática precisa voltar mais a disciplina de programação para o ensino de matemática na Educação Básica.
\end{abstract}

Palavras-chave: Programação. Formação inicial de professores. Projeto pedagógico.

\begin{abstract}
The article is the result of a research project that aimed to identify the training that future mathematics teachers have received regarding the use of technologies in the teacher education courses of Brazilian public universities. From this, in this article, we aim to understand how the initial training courses of mathematics teachers in Brazil approach the programming of computers in pedagogical projects. The research is of qualitative nature of the documentary analysis type, carried out from the analysis of pedagogical projects of the undergraduate courses in Mathematics in the years 2016 and 2017. Data analysis was based on content analysis, from which eight categories were emerged. However, in this article, we present the computer programming category and its subcategories. As results it was clear that in the PP there is a concern to connect the TDIC with the mathematical content to be taught. Despite this, there is no concern in linking programming with computational thinking, which should be stimulated in students. The initial formation of mathematics teachers needs to conduce the discipline of programming for the teaching of mathematics in Basic Education.
\end{abstract}

Keywords: Programming. Initial Teacher Training. Pedagogical Project.

\footnotetext{
${ }^{*}$ Universidade Estadual de Ponta Grossa - UEPG. Email: marcelibg@gmail.com

${ }^{* *}$ Universidade Estadual de Ponta Grossa - UEPG. Emal: prikabbaz@hotmail.com

*** Universidade Estadual de Ponta Grossa - UEPG. Emal:anabaccon@uepg.br.Bolsista Produtividade da Fundação Araucária
} 
Resumen: El artículo es fruto de un proyecto de investigación que tuvo como objetivo identificar la formación que los futuros profesores de Matemáticas han recibido acerca del uso de las tecnologías en los cursos presenciales de las universidades públicas brasileñas. A partir de eso, tenemos como objetivo comprender cómo los cursos de formación inicial de profesores de matemáticas en Brasil abordan la programación de computadoras en los proyectos pedagógicos. La investigación es de naturaleza cualitativa del tipo análisis documental, realizada a partir del análisis de proyectos pedagógicos de los cursos de Licenciatura en Matemática vigentes en el año 2016 y 2017. El análisis de los datos se basó en el análisis de contenido, del que surgieron ocho categorías. En el presente artículo, exponemos la categoría programación de computadoras y sus subcategorías. Como resultado, quedó claro que en los PP hay una preocupación de conectar las TDIC con el contenido matemático a ser enseñado. A pesar de eso, no hay una preocupación en hacer la conexión entre la programación y el pensamiento computacional, que debe ser estimulado en los alumnos. La formación inicial de profesores de matemática necesita orientar más la asignatura programación hacia la enseñanza de matemáticas en la Educación Básica.

Palabras claves: Programación. Formación Inicial de Profesores. Proyecto Pedagógico.

\section{INTRODUÇ̃̃̃O}

Nos últimos anos a sociedade contemporânea vem sofrendo transformações estruturais com o desenvolvimento das tecnologias digitais e da internet, que evoluem em uma velocidade desenfreada. Essa evolução está influenciando a vida e os costumes da sociedade, inclusive afetando diferentes camadas dela. Essa mudança tem exigido da sociedade mudanças que estão afetando o contexto educacional, que se vê com as Tecnologias Digitais da Informação e Comunicação (TDIC) adentrando seus portões e mudando o perfil do aluno. Conforme as Diretrizes Curriculares Nacionais para a formação inicial em nível superior e para a formação continuada (BRASIL, 2015) os cursos de formação inicial de professores devem estimular o uso das TDIC em diferentes estratégias de ensino aprendizagem de forma a relacionar diferentes linguagens com os meios de comunicação adotados.

Nesta direção a Base Nacional Curricular Comum (BNCC) ao considerar os critérios de relevância dos objetivos de aprendizagem e sua pertinência social na esfera do desenvolvimento dos componentes curriculares, explicita que as culturas digitais e a TDIC se constituem de temas integradores que precisam ser considerados como possíveis recursos a escola como uma ferramenta potencializadora do processo de ensino aprendizagem. (BRASIL, 2017). Visto que as crianças e os jovens estão envoltos por uma cultura digital que precisa ser explorado pelos professores, de forma ética e reflexiva no sentido de levá-los a compreender onde e como procurar informações confiáveis. A forma como a BNCC evidencia essas mudanças, traz a necessidade de reflexão sobre como estão sendo formados os futuros professores para o uso das tecnologias digitais, para que estes sejam capazes de formar também cidadãos letrados digitalmente. (BRASIL, 2017).

Todo curso de formação inicial de professores espera que os professores em formação, naturalmente transfiram os conhecimentos e habilidades adquiridas no curso em suas salas de aula. No entanto, este objetivo pode não ser alcançado no que se refere ao uso das tecnologias digitais nos processos de ensino e aprendizagem, que se caracteriza como um processo bastante complexo e multifacetado.

Sendo assim, faz-se necessário compreender como os cursos de formação inicial de professores de Matemática vêm adotando as TDIC em suas práticas de ensino aprendizagem e qual o espaço de discussão sobre o tema nas disciplinas do curso. Principalmente quando abordamos o tema de programação, uma vez que Scaico et al. (2013), Oro et al. (2015) e Garlet, Bigolin e Silveira (2018) destacam a importância desta na Educação Básica, por acreditarem que ela desenvolve habilidades diferenciadas que auxiliam no raciocínio lógico dos alunos, além de estimular a capacidade de resolução de problemas.

$\mathrm{O}$ artigo é fruto de um projeto de pesquisa que teve como objetivo identificar a formação que os futuros professores de Matemática têm recebido a respeito do uso das tecnologias nos cursos presenciais das universidades públicas brasileira. Neste sentido o objetivo deste trabalho é identificar como os cursos de formação inicial de professores de matemática no Brasil abordam a programação de computadores nos projetos pedagógicos. 


\section{APORTE TEÓRICO}

Após a publicação da atual Lei de Diretrizes e Bases da Educação Nacional em 1996, o Conselho Nacional de Educação emitiu diferentes documentos com orientações e diretrizes para os cursos de licenciatura e outros específicos para os cursos de Licenciatura em Matemática. Estes documentos previam o preparo para "o uso de tecnologias da informação e da comunicação e de metodologias, estratégias e materiais de apoio inovadores" na formação docente (CNE/CP, 2002, p. 1), sendo que esta formação inclui adquirir familiaridade desde o início do curso como instrumento de trabalho (CNE, 2001), reconhecendo o potencial destas tecnologias na resolução de problemas (CNE, 2001) e no enriquecimento da presença da prática profissional na formação do professor, o que sem dúvida nenhuma, exige a garantia da presença destes recursos, com quantidade e qualidade,nos cursos de formação (CNE/CP, 2002). Esses documentos corroboram a Sociedade Brasileira de Educação Matemática (SBEM, 2003; SBM/SBEM, 2013) quando preveem que a organização curricular dos cursos de Licenciatura em Matemática deve oferecer o preparo para o uso das TDIC enquanto atividade inerente à formação para a atividade docente.

A formação inicial de professores é o espaço no qual o futuro professor deve ser preparado para estar apto para o trabalho com TDIC no contexto de sala de aula durante as aulas de matemática. De forma a fornecer subsídios teóricos e metodológicos para que se desenvolva a relação entre as TDIC e os conteúdos matemáticos a serem ensinados. (VALENTE; ALMEIDA, 1997; SOUZA, 2016).

Os autores colocam que não é uma questão de dominar as tecnologias digitais, mas sim de compreendê-las e integrá-las ao conteúdo matemática a ser ensinado. Afinal, segundo Valente e Almeida (1997), a formação de professores deve oportunizar aos futuros professores condições deste construir seu conhecimento sobre as TDIC e prática pedagógica de ensinar matemática. Costa (2016) explica que ao compreender essa integração o futuro professor se sentirá mais seguro e apto para utilizar as TDIC na sala de aula com seus alunos. De forma a criar situações relevantes ao ensino de Matemática, proporcionando uma aprendizagem ativa na qual os alunos tem um papel mais ativo. Quando esse trabalho se realiza desde a formação inicial de professores, na busca de superar o ensino tradicional, percebe-se as TDIC como aliadas e ferramenta para o processo de ensino e aprendizagem de matemática na escola. Souza (2016) considera que é importante que a formação seja pautada na (re)construção de um conhecimento moderno integrando as TDIC.

Na busca de integrar as TDIC, os componentes e recursos tecnológicos com a escola, de forma que, estes contribuam para o processo de ensino aprendizagem, nos apoiamos no Technological Pedagogical Content Knowledge (TPACK) ou Conhecimento Tecnológico e Pedagógico do Conteúdo. Pautado em Shulman (1986; 1987) esse modelo busca definir a base de conhecimentos para o ensino, definindo aqueles necessários para que o professor possa propiciar processos de ensino e aprendizagem. Este autor explicitou várias categorias dessa base conhecimentos, que segundo Mizukami (2004) podem ser agrupadas em: conhecimento do conteúdo específico (CK), conhecimento pedagógico geral (PK) e conhecimento pedagógico do conteúdo (PCK), ou seja, analisando a estrutura proposta por Shulman e posteriormente assumida por Mishra e Koehler (2006), tem-se:

a) Conhecimento do Conteúdo (Content Knowledge - CK) - claramente, professores precisam conhecer e compreender o assunto que eles ensinam, incluindo conhecimentos dos fatos centrais, conceitos, teorias, e processos dentro de um dado campo; conhecimentos da estrutura que organiza e conecta ideias; e o conhecimento das regras das evidências e provas. (SHULMAN, 1986; 1987).

b) Conhecimento Pedagógico (Pedagogical Knowledge - PK) - é o profundo conhecimento sobre os processos de aprendizagem e práticas ou métodos de ensino e de como eles abrangem, entre outras coisas, propósitos educacionais, valores, objetivos, gerência da sala de aula, desenvolvimento e implementação do planejamento, e avaliação dos estudantes. (SHULMAN, 1986; 1987).

c) Conhecimento Pedagógico do Conteúdo (Pedagogical Content Knowledge - PCK) - é consistente com a ideia de Shulman de conhecimentos de pedagogia que são aplicáveis ao ensino de conteúdos específicos, ou seja, a transformação do conhecimento específico em conhecimento para ensinar, representação e formulação de conceitos, técnicas pedagógicas, avaliação da aprendizagem, o que faz conceitos difíceis ou fáceis de serem aprendidos, estratégias para a superação das dificuldades e confusões dos aprendizes e uma compreensão mais profunda e completa dos conceitos e conhecimentos prévios dos alunos. (SHULMAN, 1986; 1987). 
No entanto, Mishra e Koehler (2006) adicionam um novo componente a esta estrutura, o Conhecimento da Tecnologia (Technological Knowledge - TK), visando descrever os conhecimentos necessários ao professor para o uso das tecnologias nos processos de ensino e aprendizagem. Esta proposição gera novas interseções (que podem ser visualizadas na Figura 1) e que juntas compõe a estrutura TPACK.

d) Conhecimento Tecnológico (Technological Knowledge - TK) - é um conhecimento que está sempre em movimento, e assim qualquer definição pode tornar-se rapidamente ultrapassada. Esse conhecimento permite a realização de uma variedade de tarefas diferentes usando a tecnologia e a possibilidade do desenvolvimento de maneiras diferentes de realizar uma tarefa dada. Envolve as competências requeridas para operar com tecnologias específicas, que, no caso das tecnologias digitais, inclui o conhecimento da operação do sistema e da máquina, bem como a habilidade de usar um conjunto de softwares. (MISHRA; KOEHLER, 2006, 2007, 2008, 2009).

e) ConhecimentoTecnológico do Conteúdo (Technological Content Knowledge - TCK) - é o conhecimento sobre como tecnologia e conteúdo estão reciprocamente relacionados, ou seja, de que forma esse assunto pode ser modificado pela aplicação da tecnologia. (MISHRA; KOEHLER, 2006). A tecnologia enfatiza as simulações para a compreensão de fenômenos matemáticos, a grande diversidade de representação dos conceitos matemáticos e a possibilidade de navegação entre elas. Assim, o professor precisa ter uma profunda compreensão de como os conceitos ou tipos de representações podem ser modificados pelo uso da tecnologia e, baseados nesses conhecimentos, saber escolher as melhores tecnologias para a aprendizagem de um conceito específico. (MISHRA; KOEHLER, 2006, 2007, 2008, 2009).

f) Conhecimento Pedagógico da Tecnologia (Technological Pedagogical Knowledge - TPK) - conhecimento inclui a compreensão de como ensino e aprendizagem podem mudar a partir do uso de uma determinada tecnologia, saber escolher a ferramenta mais adequada, estratégias para usar o poder dessas ferramentas, conhecimento de estratégias pedagógicas e habilidade para aplicar essas estratégias no uso da tecnologia. Os professores precisam ser criativos e ter visão de como estas tecnologias podem ser utilizadas para que o aluno aprenda (MISHRA; KOEHLER, 2007, 2008, 2009).

g) Conhecimento Tecnológico e Pedagógico do Conteúdo (Technological Pedagogical Content Knowledg $e$ - TPACK) - é a base de um bom ensino com tecnologia e requer uma compreensão das representações dos conceitos usando tecnologias, das técnicas pedagógicas que usam tecnologia num caminho construtivo para ensinar o conteúdo, do que faz conceitos difíceis ou fáceis para aprender e como a tecnologia pode ajudar a corrigir alguns dos problemas enfrentados pelos alunos. A integração produtiva da tecnologia no ensino e na aprendizagem precisa considerar os três temas não isoladamente, mas antes, o complexo relacionamento desses três principais elementos entre si e com o contexto. (MISHRA; KOEHLER, 2006, 2007, 2008, 2009).

Figura 1 - As sete componentes do TPACK

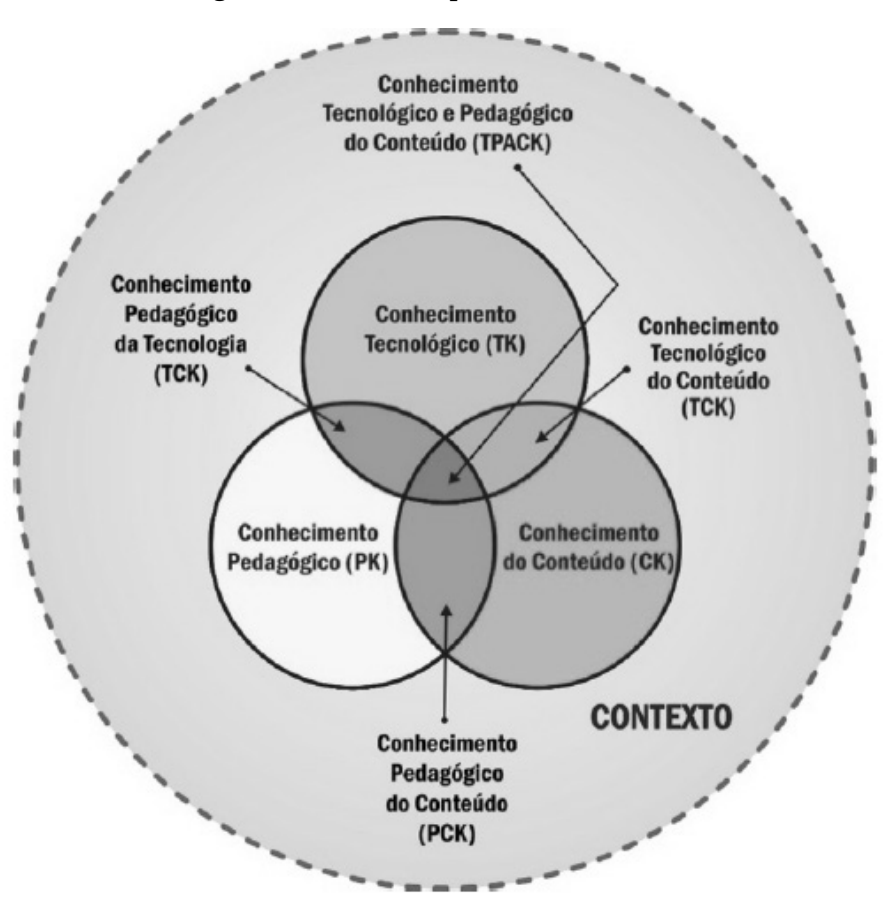

Fonte: http://www.matt-koehler.com/tpack/tpack-explained 
Mas para que o futuro professor consiga desenvolver um trabalho adequado ao processo de ensino aprendizagem de matemática mediado pelas TDIC, fazendo uso de recursos tecnológicos é necessário que está prática decorra ao longo de sua formação a fim de estimular a integração dessas no cotidiano de sua prática profissional. Em especial quando se trata de abordar conceitos computacionais desde a Educação Básica, pois conforme Scaico et al. (2013), Oro et al. (2015) e Garlet, Bigolin e Silveira (2018) a introdução da lógica de programação pode vir a auxiliar no desenvolvimento cognitivo e lógica das alunos, permitindo o desenvolvimento do pensamento computacional.

O termo computational thinking ou pensamento computacional, veio à tona com o artigo de Jeannette M. Wing, em 2006, no qual ela afirma que o pensamento computacional está relacionado ao limite e ao poder que um processo computacional possui uma vez que pode ser executado tanto por um homem como por uma máquina (WING, 2016), sendo este

[...] uma forma para seres humanos resolverem problemas; não é tentar fazer com que seres humanos pensem como computadores. Computadores são tediosos e enfadonhos; humanos são espertos e imaginativos. Nós humanos tornamos a computação empolgante. Equipados com aparelhos computacionais, usamos nossa inteligência para resolver problemas que não ousaríamos sequer tentar antes da era da computação e construir sistemas com funcionalidades limitadas apenas pela nossa imaginação. (WING, 2016, p. 4).

A programação, além de possibilitar a representação das ideias dos alunos pode também executá-las, considerando os limites da máquina e fornecendo informações que podem ser confrontadas. De forma apossibilitar que o aluno reveja conceitos, os aprimore construindo conhecimentos, ou seja, as ações que mais contribuem para o processo de construção de conhecimento são certamente a reflexão e a depuração. As mesmas acontecem a partir de uma resposta que o aprendiz obtém da execução da descrição da resolução do problema, fornecida em termos de comandos de um determinado software. (VALENTE, 2002, 2009).

Neste processo o papel do professor é central, pois algumas vezes o aluno não dispõe de conhecimento suficiente para progredir, o que pode levar ao abandono do ciclo de aprendizagem. Entra em cena o professor, que tem a função de manter o aluno na espiral. Para tanto, o professor pode explicitar o problema, conhecer o aluno e como ele pensa, incentivar e desafiar para diferentes níveis de descrição, trabalhar com diferentes níveis de reflexão, contribuir com a depuração.

Dessa forma voltamos nossas lentes para os projetos pedagógicos dos cursos - PPC de licenciatura em matemática, pois este é:

O documento orientador de um curso que traduz as políticas acadêmicas institucionais. Entre outros elementos, é composto pelos conhecimentos e saberes necessários à formação das competências estabelecidas a partir do perfil do egresso; estrutura e conteúdo curricular; ementário; bibliografia básica e complementar; estratégias de ensino; docentes; recursos materiais; laboratórios e infra-estrutura de apoio ao pleno funcionamento do curso. (BRASIL, 2008, p. 24)

Conforme Masetto (2012) o PP consiste na organização interna do curso em uma Instituição de Ensino Superior - IES. No qual se apresenta como um todo orgânico e articulado que busca através dos objetivos e metas, ligar professores, alunos, disciplinas, carga horária, recursos entre outros. Afinal é neste documento que as Instituições de Ensino Superior (IES) explicitam suas concepções de formação inicial, de forma a interligar o PP com o Projeto pedagógico Institucional (PDI). (BRASIL, 2015). Isso auxilia na definição do perfil do profissional que o curso pretende formar, sua visão de sociedade além de definir os projetos que se pretende desenvolver. (MASETTO, 2012).

\section{OS CAMINHOS METODOLÓGICOS ADOTADOS NA PESQUISA}

Este artigo aborda alguns resultados de uma pesquisa que permitiu compreender a presença da TDIC nos Projetos Pedagógicos dos cursos de Licenciatura em Matemática de IES públicas brasileiras. A pesquisa tem uma abordagem qualitativa visto que analisar e refletir a realidade "através da utilização 
de métodos e técnicas para compreensão detalhada do objeto de estudo em seu contexto histórico e/ou segundo sua estruturação.” (ANDRÉ, 2005, p. 41). Essa pesquisa apesar de adotar a pesquisa qualitativa fará uso de dados quantitativos com objetivo de compreender o espaço das TDIC nos projetos pedagógicos dos cursos de Licenciatura em Matemática. Adotou-se a pesquisa documental pautando-a nos Projetos Pedagógicos - PP dos cursos de Licenciatura em Matemática vigentes no ano de 2016 e 2017, como fonte de pesquisa.

Para a coleta de dados foi acessado o site de cada instituição a fim de encontrar o projeto pedagógico dos cursos, e em alguns casos recorremos também as ementas das disciplinas. Para a delimitação dos cursos e dos projetos pedagógicos a comporem o corpus desta pesquisa, definiu-se primeiramente que trabalharíamos com as diferentes regiões brasileiras, estabelecendo assim os seguintes critérios:

a) ser um curso de instituição pública;

b) ofertado na modalidade presencial;

c) com grau de Licenciatura em Matemática;

d) que esteja ativo no Banco de Dados sobre o Ensino Superior - e-MEC.

Uma dificuldade encontrada foi relativa às IES que não explicitavam se o curso era presencial ou a distância, por este motivo resolve-se excluir estas instituições. Além disso, foi necessário excluir as ofertas referentes a Licenciatura em Ciências Naturais e Matemática, Licenciatura em Ciências Matemática e Física, Licenciatura em Educação Indígena com Habilitação em Física e Matemática, cursos ofertados pelo Programa Nacional de Formação de Professores da Educação Básica (Parfor), cursos presenciais mediados ou modulares, Matemática com ênfase em Informática, Matemática, Informática e Tecnologias, uma vez que não atendiam aos critérios estabelecidos.

Após a identificação das IES, as mesmas foram separadas conforme a região e pelo tipo de instituição, sendo elas: universidades federais, universidades estaduais e institutos federais. O tamanho da amostra foi definido em $10 \%$ da população. A sua composição foi estratificada em relação a região e também em relação ao tipo de instituição pública (federal, estadual ou instituto federal), sendo necessário incluir uma instituição de cada região na amostra, conforme tabela 1, a fim de analisar pelo menos uma instituição de cada tipo (federal, instituto e estadual).

Quadro 1: Estratificação da amostra por região e tipo de instituição.

\begin{tabular}{|c|c|c|c|c|c|c|c|c|}
\hline Região & \multicolumn{2}{|c|}{ Federal } & \multicolumn{2}{c|}{ Instituto } & \multicolumn{2}{c|}{ Estadual } & \multicolumn{2}{c|}{ Total } \\
\hline & $\begin{array}{c}\mathrm{N}^{\circ} . \\
\text { IES }\end{array}$ & $\begin{array}{c}\mathrm{N}^{\circ} . \\
\text { Amostra }\end{array}$ & $\begin{array}{c}\mathrm{N}^{\circ} . \\
\text { IES }\end{array}$ & $\begin{array}{c}\mathrm{N}^{\circ} . \\
\text { Amostra }\end{array}$ & $\begin{array}{c}\mathrm{N}^{\circ} . \\
\text { IES }\end{array}$ & $\begin{array}{c}\mathrm{N}^{\circ} . \\
\text { Amostra }\end{array}$ & $\begin{array}{c}\mathrm{N}^{\circ} . \\
\text { IES }\end{array}$ & $\begin{array}{c}\mathrm{N}^{\circ} . \\
\text { Amostra }\end{array}$ \\
\hline SUL & 8 & 1 & 16 & 2 & 15 & 2 & 39 & 5 \\
\hline CENTRO- OESTE & 12 & 2 & 5 & 1 & 16 & 2 & 33 & 5 \\
\hline NORTE & 16 & 2 & 8 & 1 & 9 & 2 & 33 & 5 \\
\hline SUDESTE & 25 & 3 & 24 & 3 & 16 & 3 & 75 & 9 \\
\hline NORDESTE & 34 & 4 & 28 & 3 & 32 & 3 & 89 & 10 \\
\hline
\end{tabular}

Fonte: Dados da pesquisa, 2016-2017.

O tamanho da amostra foi definido em $10 \%$ da população. A sua composição foi estratificada em relação a região e também em relação ao tipo de instituição pública (federal, estadual ou instituto federal), sendo necessário incluir uma instituição de cada região na amostra, conforme tabela 1 acima, a fim de analisar pelo menos uma instituição de cada tipo (federal, instituto e estadual). A definição das IES que teriam o Projeto Pedagógico - PP analisado foi realizado através de sorteio, assim, quando duas instituições do mesmo estado eram sorteadas, um novo sorteio era realizado, ficando a amostra definida conforme o quadro 2 . 
Quadro 2 - Amostra por região e tipo de instituição - Cursos de licenciatura em matemática de IES públicas brasileiras cujo PP explicita sobre a EaD

\begin{tabular}{|c|c|c|c|c|c|c|c|c|c|c|}
\hline is & \multicolumn{10}{|c|}{ INSTITUIÇÕES DE ENSINO SUPERIOR } \\
\hline 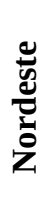 & 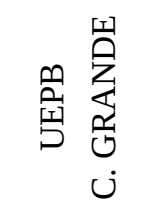 & 空 & 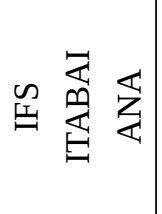 & 空悹 & 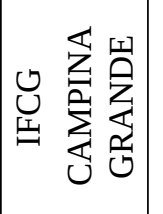 & 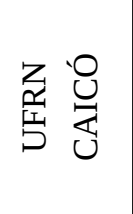 & 芯导 & 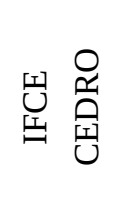 & 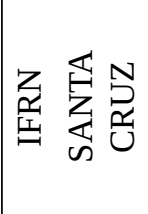 & 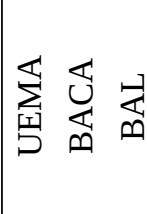 \\
\hline 苞 & 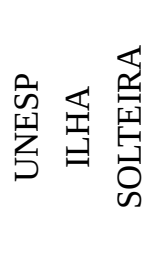 & 暒它 & 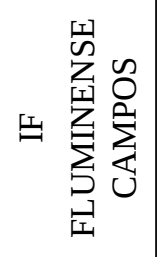 & 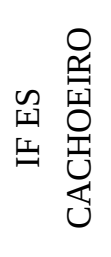 & 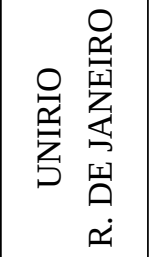 & 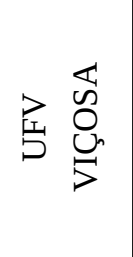 & 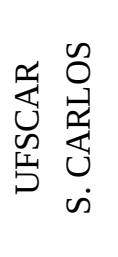 & 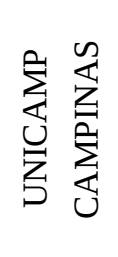 & 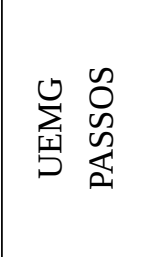 & \\
\hline $\bar{E}$ & \multicolumn{2}{|c|}{$\begin{array}{c}\text { FECILCAM } \\
\text { CAMPO MOURÃO }\end{array}$} & \multicolumn{2}{|c|}{$\begin{array}{l}\text { UDESC } \\
\text { JOINVILE }\end{array}$} & \multicolumn{2}{|c|}{$\begin{array}{l}\text { UNIPAMPA } \\
\text { ITAQUI }\end{array}$} & \multicolumn{2}{|c|}{$\begin{array}{c}\text { IFRS } \\
\text { IBIRUBÁ }\end{array}$} & \multicolumn{2}{|c|}{$\begin{array}{c}\text { IF FARROUPILHA } \\
\text { SÃO BORJA }\end{array}$} \\
\hline 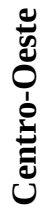 & \multicolumn{2}{|c|}{$\begin{array}{c}\text { UEMS } \\
\text { CASSILÂNDIA }\end{array}$} & \multicolumn{2}{|c|}{$\begin{array}{c}\text { UFMS } \\
\text { TRES LAGOAS }\end{array}$} & \multicolumn{2}{|c|}{$\begin{array}{c}\text { UFMT } \\
\text { RONDONÓPOLIS }\end{array}$} & \multicolumn{2}{|c|}{$\begin{array}{c}\text { IFMT } \\
\text { PARECIS }\end{array}$} & \multicolumn{2}{|c|}{$\begin{array}{l}\text { UNEMAT } \\
\text { BARRA DOS } \\
\text { BUGRES }\end{array}$} \\
\hline : & \multicolumn{2}{|c|}{$\begin{array}{l}\text { UEPA } \\
\text { BELEM }\end{array}$} & \multicolumn{2}{|c|}{$\begin{array}{c}\text { UFRR } \\
\text { BOA VISTA }\end{array}$} & \multicolumn{2}{|c|}{$\begin{array}{l}\text { UFPA } \\
\text { BELÉM }\end{array}$} & \multicolumn{2}{|c|}{$\begin{array}{c}\text { IFTO } \\
\text { PALMAS }\end{array}$} & \multicolumn{2}{|c|}{$\begin{array}{c}\text { UERR } \\
\text { BOA VISTA }\end{array}$} \\
\hline
\end{tabular}

Fonte: Dados da pesquisa, 2016-2017.

Para a realização da análise dos documentos partiu-se dos PP do curso de Licenciatura em Matemática das IES sorteadas e/ou ementa das disciplinas do curso. Optou-se por desconsiderar as disciplinas optativas de cada curso. Com as ementas e/ou PP utilizou-se a ferramenta de busca de palavras do Acrobat Reader, para a busca dos seguintes termos: tecnologia, computa, TIC, TDIC, computa, recurso, EaD, distância, programação, informática, software, aplicativo, internet, web, rede, mídia, applet, numérico, simula, dinâmic, móvel. De posse dos dados optou-se por pautar a análise dos dados na análise de conteúdo (BARDIN, 2007), da qual emergiram oito categorias: Categoria I: tecnologias assistivas; Categoria II: educação à distância; Categoria III: programação de computadores; Categoria IV: desenvolvimento de conceitos matemáticos com o uso das TDIC; Categoria V: objetivos do curso e perfil do egresso; Categoria VI: exploração de softwares de uso geral e específicos da Matemática; Categoria VII: TDIC articuladas com a prática; Categoria VIII: formação específica de professores de Matemática para o uso das TDIC em processos de ensino e aprendizagem.

Entretanto, buscando alcançar o nosso objetivo, no presente artigo optamos por apresentar e analisar apenas a categoria III, relativa à presença da programação de computadores. A partir da análise dessa categoria foi possível identificarmos quatro subcategorias nos PP dos cursos de Licenciatura em Matemática sendo eles: (a) oferta de uma disciplina totalmente voltada para a programação de computadores; (b) programação de computadores em disciplinas voltadas para o ensino; (c) programação de computadores em outras disciplinas voltadas para conceitos matemáticos; e (d) programação de computadores e cálculo numérico. 


\section{UMA ANÁLISE DA CATEGORIA - PROGRAMAÇÃO DE COMPUTADORES}

A fim de compreendermos como a disciplina de programação de computadores vem sendo ofertado nos PP, como resultados da pesquisa, nessa seção passamos apresentar a análise da Categoria Programação de computadores, aqui representada pelas suas quatro subcategorias e sua incidência na amostra analisada. Essa categoria reúne as unidades de análise relacionadas à Programação de Computadores, ou seja, como cada um dos PP tem organizado as suas disciplinas relacionadas a essa temática.

Em relação à primeira subcategoria denominada oferta de uma disciplina totalmente voltada para a programação de computadores, foi possível verificar nos PP que há disciplina(s) totalmente voltada(s) para a programação de computadores, ou disciplina(s) que exploram a programação de computadores como um conteúdo. Ao analisarmos os PP foi possível verificar que 55,9\% das IES oferecem uma disciplina voltada para a programação de computadores.

Se observamos esse número distribuído entre as regiões é possível verificar que a região Norte, é a região com maior índice, proporcionalmente, de instituições que não oferecem disciplina específica voltada para a programação (3 cursos), seguida das regiões nordeste (5 cursos), Sul e Centro-Oeste (2 cursos cada uma), com o menor índice a região Sudeste (3 cursos). O que se pode inferir a partir desses números é que a região Sudeste por ser, segundo o Instituto Brasileiro de Geografia e Estatística - IBGE. (IBGE, 2015), uma das regiões que tem muitos usuários da internet com celulares, seguida da região Sul e Centro-Oeste. Esses números elevados podem contribuir para a compreensão de que a disciplina voltada para a programação de computadores faz-se necessária em um curso de formação inicial de professores de matemática. Outro ponto levantado por Garlet, Bigolin e Silveira (2018) é a necessidade de profissionais capacitados para trabalhar o ensino da programação, na realidade escolar da Educação Básica, visto que para os autores a computação envolve diversas áreas de conhecimento e atuação profissional. Esses dados abrem questionamentos sobre as contribuições da programação para a formação do professor de Matemática, e em última instância para os alunos da Educação Básica. Afinal:

[...] a ideia não é ensinar uma linguagem de programação específica, como Java, C++, PHP ou qualquer outra, mas sim ensinar a lógica que é a mesma para todas as linguagens. Assim, se $o$ aluno souber a lógica ele terá grande facilidade de aprender a sintaxe da linguagem. Cabe destacar que nem todos os alunos se tornarão programadores, eles vão optar por outras áreas de conhecimento, porém com um diferencial, terão maior capacidade de pensar e com mais criatividade, pois é isso que a aprendizagem da lógica de programação faz, desenvolve várias habilidades que muitas vezes estão ocultas. (GARLET, BIGOLIN, SILVEIRA, 2018, p. 2).

Esta argumentação tem promovido mudanças na compreensão do papel da programação, ou seja, numa preocupação que ultrapassa o aprender a programar, mas no sentido de criar condições de desenvolver o pensamento computacional, ou seja, a programação poderia ajudar a pensar melhor. (VALENTE, 2016).

Na análise dos PP dos cursos, a programação é entendida como atividade de fornecer instruções ao computador a fim de resolver um conjunto de problemas, e estão centradas estritamente na programação de computadores e não se menciona o desenvolvimento do pensamento computacional, como é possível observar nas ementas que seguem.

Introdução à Programação I: Elementos de uma linguagem algorítmica. Comandos básicos da linguagem. Algoritmos x programas. Tipos estruturados de dados. Subprogramas. Técnicas básicas de programação. (UFV, 2013, p. 47).

Lógica de Programação: Conceito de lógica de programação e algoritmos; Estrutura sequencial, condicional e de repetição; Estrutura de dados; Modularização. (IFCE, 2012, p. 157).

Algoritmo de Linguagem de Programação: Noções básicas sobre sistemas de computação. Noções sobre linguagens de programação e programas. Estudo de uma linguagem de alto nível. (UDESC, 2005, p. 25).

No entanto, não é possível afirmar que tais disciplinas não contribuam para o desenvolvimento do pensamento computacional. Já que segundo Valente (2016), há autores que defendem que a criatividade e o pensamento computacional são desenvolvidas em atividades centradas estritamente na programação 
de computadores; por outro lado, há autores que defendem que o pensamento computacional não é exclusivamente desenvolvido pela programação de computadores. A programação por si só não é suficiente para promover o desenvolvimento do pensamento computacional, ou seja, o pensamento computacional deve contribuir para que cada pessoa possa compreender como usar a computação para resolver os seus problemas, para criar e para descobrir novas questões que podem ser exploradas produtivamente. Pensando na possibilidade de o pensamento computacional contribuir com a programação.

Um fato que nos chamou a atenção é que na Universidade Estadual de Campinas - Unicamp as ementas diferem para o curso diurno e o noturno, isso se deve ao fato de que o diurno mais conhecido como "Cursão", é um curso oferecido pelo Instituto de Matemática, Estatística e Computação Científica (IMECC) e pelo Instituto de Física GlebWataghin (IFGW), que permite ao acadêmico cursar disciplinas da área de exatas, deixando para decidir posteriormente qual será a sua carreira que pode ser no bacharelado ou na licenciatura. Dessa forma as ementas diferem, como pode-se verificar abaixo:

\begin{abstract}
Algoritmos e Programação de Computadores - Licenciatura Diurno:Conceitos básicos de organização de computadores. Construção de algoritmos e sua representação em pseudocódigo e linguagens de alto nível. Desenvolvimento sistemático e implementação de programas. Estruturação, depuração, testes e documentação de programas. Resolução de problemas. Programa: Introdução à computação. Algoritmos e programas. Variáveis e Atribuições. Comandos condicionais. Comandos de Entrada e Saída. Comandos repetitivos. Procedimentos e funções. Vetores e matrizes. Recursão. Arquivos. Registros. Memória dinâmica. Outros tópicos. (UNICAMP, 2015, p. 150).
\end{abstract}

Introdução à Matemática Computacional - Licenciatura Noturno: Introdução a algoritmos em uma linguagem de programação voltada para Matemática: variáveis, condicionais, laços e funções. Representação de números em ponto flutuante e noções de erros numéricos. Zeros de funções. Métodos diretos para a solução de sistemas lineares. Quadrados mínimos lineares. Interpolação. Programa: Introduzir os fundamentos de matemática computacional e computação científica, com ênfase em métodos numéricos básicos utilizados na solução de problemas matemáticos; promover a utilização de pacotes computacionais; analisar a influência dos erros introduzidos na utilização e implementação computacional destes métodos. (UNICAMP, 2015, p. 155).

Como podemos perceber a ementa do curso diurno é mais voltado a programação em si, enquanto a ementa do curso noturno associa a programação ao ensino do Cálculo Numérico, mostrando mais a aplicação desse conhecimento. Vemos que não há a preocupação de oportunizar aos futuros professores a formação do pensamento computacional. Mas é inegável que a ementa do curso noturno é mais aplicada e busca a resolução de problemas a partir da programação. Pensando na possibilidade de o pensamento computacional contribuir com a programação, Valente (2016) afirma que, há autores que defendem que, para ampliar e garantir sucesso em atividades de programação é preciso explorar inicialmente as bases do pensamento computacional, com atividades relacionadas ao pensamento computacional. De forma a desenvolver a linguagem computacional através de atividades em que o aluno possa expor esse pensamento computacional. (VALENTE, 2016).

De forma a preparar melhor os futuros professores não só na compreensão da programação ou mesmo do pensamento computacional, mas sim de como integrar esses conhecimentos no ensino de matemática na Educação Básica.

Nesta categoria podemos identificar uma forte ênfase no Conhecimento Tecnológico (Technological Knowledge - TK), que é um dos pilares da estrutura TPACK. Não podemos afirmar que os futuros professores conhecem programação, já que não se pode ter certeza que apenas uma disciplina de programação no curso Licenciatura em Matemática garanta o seu domínio, oportunize a compreensão do funcionamento mais profundo das tecnologias, que ultrapassa o simples apertar de teclas. Este conhecimento abre um leque de possibilidades não só de consumo das tecnologias, mas de modificação e criação de outras.

A segunda subcategoria é a programação de computadores em disciplinas voltados para o ensino, ela foi abordada em 4 instituições que elencam em seu PP uma disciplina que relacionem a programação e a formação de professores para a Educação Básica. Garlet, Bigolin, Silveira (2018) destacam a 
importância que há entre a integração da programação com o ensino na Educação Básica, uma vez que estes podem despertar o interesse dos alunos e diminuir a evasão dos cursos. Afinal os futuros professores podem desenvolver o pensamento cognitivo e mudar o seu papel no processo de ensino aprendizagem.

Informática no ensino da matemática: introdução à computação. Noções de programação em computadores, linguagens e softwares específicos para uso no ensino de matemática. (UEMS, 2011, p. 34).

Informática aplicada à educação II:introdução à linguagem de programação. Comandos e bloco de comandos. Arquivos e análise de dados. Rotinas gráficas. Construção de algoritmos para prática pedagógica. Informática aplicada à sala de aula. (IFBA, 2014, p. 74)

Ferramentas computacionais para o ensino da matemática: utilização das novas tecnologias no processo de ensino/aprendizagem de matemática. Abordagem teórico-prática sobre o uso do computador, da internet e da tecnologia digital. Implicações pedagógicas relativas ao uso da tecnologia digital no processo de ensino /aprendizagem da matemática. Ferramentas computacionais para o ensino e aprendizagem da matemática. Planilhas eletrônicas. Ferramentas para computação algébrica. Ferramentas para computação numérica. Ferramentas para o ensino de geometria. Ferramentas para o ensino de lógica-matemática. Produção de conteúdo para internet. (UFRN, 2010, p. 59)

Novas tecnologias aplicadas ao ensino de matemática: A integração da multimídia no Currículo de Matemática da Educação Básica: caracterização, usos consolidados e desafios. Avaliação de softwares educacionais para a Educação Matemática: principais usos em sala de aula e novas possibilidades de aplicações. Tecnologias interativas como ferramentas para a Educação Matemática: Ferramentas Web, Ambientes Virtuais para Educação a Distância. A Educação Matemática com Programação e Robótica. Ferramentas tecnológicas de conteúdo específico: Sistemas de computação algébrica simbólica; ambientes de geometria dinâmica; applets interativos. (UNESP, 2014, p. 39).

Nas duas primeiras disciplinas, ofertadas respectivamente pela UEMS e IFBA, verificamos que há articulação entre a programação e a formação de professores para a Educação Básica, apesar de issosó acontecer no título das disciplinas, visto que os conteúdos da ementa abordam questões de programação e somente um conteúdo que se aproxima mais do ensino. Não é possível afirmar só com base na ementa que as disciplinas não estimulem essa relação de forma mais clara. Já as duas últimas, ofertadas respectivamente pela UFRN e pela UNESP, ficou explícito que há uma preocupação mais evidente, nos conteúdos da ementa, com a formação deste profissional visando sua atuação no ensino de Matemática com tecnologia. Estas disciplinas tem a preocupação de capacitar os futuros professores no uso de ferramentas que possam contribuir para o processo de ensino aprendizagem do pensamento computacional fazendo uso de recursos tecnológicos diferenciados e adequados a faixa etária, de forma a tornar mais fácil a aprendizagem da programação envolvendo o aluno e buscando despertar o interesse dele. (GARLET; BIGOLIN; SILVEIRA, 2018).

Analisando os conhecimentos necessários ao professor para o uso das tecnologias no ensino da Matemática, esta categoria teria a potencialidade mais explícita de articular a componente pedagógica da estrutura TPACK às demais componentes envolvidas na programação, compreendendo que não se trata apenas da inclusão de uma dimensão, mas também da inclusão das suas interseções com os demais conhecimentos previstos nesta estrutura.

A terceira subcategoria referente aprogramação de computadores em outras disciplinas voltadas para conceitos matemáticos, encontramos somente três PP que estabelecem essa relação, contemplando a interseção da programação com outros conhecimentos matemáticos, tais como Teoria dos Números (UFAL, 2007; UFSCAR, 2007) e Álgebra Linear (IF ES, 2010).

Introdução à teoria dos números: História da Aritmética e da Teoria dos Números. Sistemas de representações numéricas e operações aritméticas. Divisibilidade, mdc, mmc. Números primos e o Teorema Fundamental da Aritmética. Equações diofantinas lineares. Introdução às congruências e aplicações. Algoritmos computacionais aplicados à Teoria dos Números. (UFSCAR, 2007, p. 29) 
Introdução à teoria dos números: Estudo da aritmética e sua relação com a cultura dos povos. Compreender a relação do desenvolvimento dos sistemas de numeração com o progresso cultural e científico. Perceber a importância da presença da Aritmética nas escolas fundamental e média. Flexibilizar o estudo tradicional da Aritmética e dos conceitos iniciais da Teoria dos Números, usando tanto os métodos da Álgebra quanto os da Matemática Discreta (algoritmos). Dar oportunidade para o estudante adquirir confiança pessoal em desenvolver atividades matemáticas. Vivenciar a Arte de Investigar em Matemática tendo como substrato a Aritmética e a Teoria dos Números. Propiciar a vivência da criatividade, iniciativa e trabalho coletivo. Conteúdos tratados: História da Aritmética e da Teoria dos Números. Sistemas de representações numéricas e operações aritméticas. Divisibilidade, mdc, mmc. Números primos e o Teorema Fundamental da Aritmética. Equações diofantinas lineares. Introdução às congruências e aplicações. Algoritmos computacionais aplicados à Teoria dos Números. Alguns teoremas clássicos. (UFAL, 2007, p. 21-22.)

Álgebra linear: Geral: Proporcionar ao estudante uma visão integrada dos conceitos de Álgebra Linear e suas aplicações, tornando o estudante capaz de reconhecer e resolver problemas na área, associados a futuras disciplinas e/ou outros projetos a que se engajarem. Específicos: Demonstrar a importância da álgebra linear nos recentes avanços tecnológicos no trabalho com vetores “n-dimensionais” num espaço além do visual (tridimensional) com ênfase na sua representação matricial. Indicar a utilização de técnicas computacionais em problemas trabalhosos que norteiam disciplinas como Programação linear, Pesquisa Operacional, Engenharia Elétrica, Computação Gráfica, Teoria dos Fractais, Criptografia etc. (IF ES, 2010, p. 94).

Conforme Garlet, Bigolin, Silveira (2018) a lógica de programação deve estar ligada a outras disciplinas, neste caso vemos a ligação com disciplinas de conteúdo específico da matemática, o que pode contribuir para a compreensão do futuro professor a respeito dos conteúdos estudados.

A quarta subcategoria, que aborda a programação de computadores e o Cálculo Numérico, logicamente é contida na categoria anterior, mas optou-se em analisá-la separadamente, uma vez que o conjunto de conceitos matemáticos que envolvem Cálculo Numérico possuem uma relação muito estreita com a programação computacional.

Dos 34 PP de cursos de Licenciatura em Matemática analisados, 3 não apresentam a disciplina de Cálculo Numérico eem 5 ela está prevista como disciplina eletiva. Estes dados no levam a questionar sobre a importância da presença dessa temática em cursos de formação inicial de professores de Matemática.

Segundo as reflexões produzidas pela Sociedade Brasileira de Matemática (SBM) em parceria com a Sociedade Brasileira de Educação Matemática (SBEM) acerca da formação do professor de Matemática no curso de Licenciatura, é recomendável que o futuro professor desenvolva a capacidade de aprender e aplicar as novas técnicas de Matemática Aplicada, diante da presença cada vez maior dos avanços que só foram alcançados pela utilização da modelagem matemática e dos métodos numéricos, ou seja,

O conhecimento dos métodos de cálculo numérico prepara melhor o licenciando de matemática para sua prática pedagógica, reforçada pela necessidade de contextualizar os problemas abordados em sala de aula, que demandam o domínio de princípios de modelagem matemática e da natureza de números reais. (SBM/BEM, 2013, p. 33).

A disciplina de cálculo numérico quando abordada com diferentes metodologias de ensino, pode proporcionar ao futuro uma melhor compreensão dos conteúdos estudados e os permite utilizar ferramentas que auxiliam na "[...] solução de problemas que o cálculo analítico, muitas vezes, não consegue solucionar ou que são de difícil resolução.” (GAMA; GOMES; PIRES, 2018, p. 239).

No entanto, nesta subcategoria o foco foi investigar se os PP preveem a relação entre esta disciplina e a programação computacional. Sobre esta relação, é importante observar que segundo a SBM/SBEM (2013) a utilização de ferramentas computacionais pode ser oportuna para o ensino aprendizagem de métodos numéricos, oportunizando o conhecimento e domínio de softwares que auxiliem na testagem das conjecturas, validações e resoluções de problemas. No entanto, dos 27 cursos em que o Cálculo Numérico aparece como disciplina obrigatória (79,41\% da amostra), em apenas 9 cursos de Licenciatura em Matemática o PP deixa evidente esta relação entre a disciplina de Cálculo Numérico e a programação de computadores. Esta relação aparece nos objetivos das disciplinas em 7 cursos de licenciatura, como: 
- Apresentar ao aluno as primeiras noções de métodos de obtenção de soluções aproximadas de problemas de cálculo e de álgebra linear, através de algoritmos programáveis. Prover soluções aproximadas de problemas cuja solução exata é inacessível. (UFSCAR, 2005, p. 31).

- Conhecer métodos numéricos utilizados na resolução de problemas matemáticos com o auxílio da computação; analisar a adequação do(s) método(s) numérico(s) empregado(s) aos diversos tipos de problemas e identificar as inconveniências e vantagens que cada método oferece. (UEMS, 2011, p. 38).

- Estudar técnicas numéricas de solução de expressões matemáticas. Apresentar os conceitos matemáticos do cálculo numérico requeridos na área de engenharia, ressaltando as fundamentações teóricas, vantagens e dificuldades Computacionais dos métodos numéricos. (IFES, 2010 p. 107)

- Interpretar e analisar questões relacionadas aos métodos numéricos e resolvê-las utilizando calculadoras e softwares. (IF FLUMINENSE, 2012, p. 47-50).

- Conhecer as ligações orgânicas entre a origem da Matemática e a computação. Conhecer e analisar as demonstrações formais. Analisar o grande número de problemas interessantes que não requerem ferramentas sofisticadas para a sua compreensão. Realizar estudos sobre o processo das construções de algoritmos. Entender os fundamentos teóricos dos métodos numéricos usados por computadores na execução dos diversos cálculos matemáticos. (IFCE CEDRO, 2012, p. 162).

- Apresentar técnicas numéricas computacionais para resolução de problemas nos campos das ciências e da engenharia, levando em consideração suas especificidades, modelagem e aspectos computacionais vinculados a essas técnicas. No final de cada tópico, o aluno deve ser capaz de selecionar a técnica numérica computacional mais adequada para tratar o problema a ser resolvido e aplicar a técnica manualmente ou com o uso de computador e analisar os resultados obtidos. (IF SP, 2012, p. 70)

- Introduzir os fundamentos dos métodos numéricos básicos utilizados na solução de problemas matemáticos que aparecem comumente nas engenharias e ciências aplicadas; promover a utilização de pacotes computacionais; analisar a influência dos erros introduzidos na utilização e implementação computacional destes métodos. (UNICAMP, 2015, p. 154).

Somente uma das IES adota em seu PP o uso de competências e habilidade de forma que a disciplina de Cálculo Numérico é abordada da seguinte forma:

Competência: Utilizar correta e adequadamente instrumentos de medição e recursos tecnológicos como meios de resolução de situações-problema. Habilidades: Resolver problemas físicos utilizando algoritmos especializados e máquinas digitais. (IFTO, 2015, p. 130-133).

A última instituição incluída nesta categoria é o Instituto Federal de Educação, Ciência e Tecnologia do Rio Grande do Sul, Campus Ibirubá, que prevê esta articulação entre Cálculo Numérico e programação na própria ementa da disciplina

Erros. Solução de equações algébricas e transcendentes. Solução de equações polinomiais. Solução de sistemas e equações lineares. Interpolação e diferenciação. Ajuste de curvas. Integração numérica. Solução numérica de equações diferenciais. Estudo de construção de algoritmos e implementação em linguagem de Programação. (IFRS, 2014, p. 74)

Pode-se verificar nos objetivos propostos que há uma preocupação de aliar o Cálculo Numérico a resolução de problemas, pois conforme Gama, Gomes e Pires (2018) ao relacionar o conteúdo com o cotidiano dos alunos torna a aprendizagem mais significativa que pode levar o futuro professor a desenvolver a reflexão e a criação de ações e estratégias. Outro ponto que se evidencia nos objetivos é o incentivo ao uso das TDIC com o intuito de facilitar a aprendizagem dos conteúdos de Cálculo Numérico, além de adotar metodologias diferenciadas que auxiliam a minimizar os problemas de aprendizagem. Somente o IFCE Cedro (2012) tem como objetivo estabelecer a relação entre a matemática e a computação e suas diferentes linguagens conforme Garlet, Bigolin e Silveira (2018).

Analisando a terceira e a quarta subcategoria à luz da estrutura TPACK é possível inferir um forte potencial de desenvolvimento do Conhecimento Tecnológico do Conteúdo (Technological Content Knowle 
dge - TCK) nessas componentes curriculares dos cursos de Licenciatura em Matemática. Essa contribuição pode se dar no sentido de analisar as contribuições da Matemática para o surgimento e desenvolvimento dos computadores como das influências que a Matemática recebe das tecnologias. Exemplo dessas contribuições da informática para a Matemática, enquanto ciência em desenvolvimento, foram citados por Ponte e Canavarro (1997): despertar da álgebra linear (em especial a teoria das matrizes), relançou o estudo de métodos iterativos já abordado por matemáticos como Euler e Gauss, grande desenvolvimento de certos domínios de investigação como a análise numérica, a lógica e as estruturas discretas abstratas, estimulo ao desenvolvimento de novas áreas como a teoria do caos, os fractais, a programação linear e não linear e o estudo da complexidade computacional.

Segundo Ponte e Canavarro (1997) o computador também se constitui em uma forte influência no desenvolvimento da Matemática atual, levando ao estabelecimento de um novo paradigma de investigação, a Matemática experimental. Nesta perspectiva, o autor cita a possibilidade trazida pelo computador de realizar experiências, testando conjecturas que envolvem grande quantidade de cálculos e a estratégia proporcionada pelo computador de decompor um problema num grande número de casos especiais, usando-o depois para verificar, um a um, esses casos todos. Ambas possibilidades seriam impossíveis pelos processos tradicionais.

\section{CONSIDERAÇÕES FINAIS}

No presente artigo, tivemos como objetivo identificar como os cursos de formação inicial de professores de Matemática no Brasil abordam a programação de computadores nos projetos pedagógicos. A partir da análise da categoria 'programação de computadores' e suas subcategorias, - programação de computadores, foi possível identificarmos que há um alto índice de instituições nas regiões Norte, Sul e Nordeste que não oferecem nenhuma disciplina, total ou parcialmente, voltada para a programação de computadores. Nas instituições que oferecem tal disciplina, a programação é entendida como atividade de fornecer instruções ao computador, e estão centradas estritamente na programação de computadores e não se menciona o desenvolvimento do pensamento computacional, o que não permite afirmar que tais cursos não estejam explorando o pensamento computacional em outras disciplinas.

A partir da literatura, o que se pode concluir é que, o pensamento computacional é mais amplo que a atividade de programação, já que o mesmo é útil para outras atividades. Por outro lado, a programação contribui para o seu desenvolvimento. Neste sentido, seria interessante conhecer as contribuições que se espera que tais disciplinas tragam na formação de professores de Matemática: se para o desenvolvimento do pensamento computacional do futuro professor, no sentido de que ele vivencie situações de programação em que tal pensamento é utilizado e seja um profissional capaz de proporcionar atividades que desenvolvam este pensamento em seus alunos, ou se apenas visando uma capacitação para cursar outras disciplinas do próprio curso ou da pós-graduação.

De qualquer forma, diante da realidade deste movimento do pensamento computacional na Educação Básica em diversos países, baseada no argumento de que até aqui as TDIC tiveram uma contribuição restrita na educação e pouco contribuíram na forma de pensar e resolver problemas, e que o pensamento computacional é um conhecimento fundamental no contexto da cultura digital, para viver e atuar na sociedade do conhecimento. (VALENTE, 2016). É preciso que os cursos de licenciaturas, e aqui não só as licenciaturas em Matemática, promovam discussões sobre o impacto da utilização da tecnologia no desenvolvimento cognitivo, ou seja, na mente, e que esta discussão traga reflexões que orientem as futuras decisões curriculares para a formação de professores.

Em relação ao desenvolvimento do TPACK, a programação nas duas manifestações dominantes nos PP dos cursos de Licenciatura em Matemática do Brasil, traz uma forte contribuição no desenvolvimento do Conhecimento Tecnológico (Technological Knowledge - TK) e do Conhecimento Tecnológico do Conteúdo (Technological Content Knowledge - TCK), por meio de disciplinas de totalmente voltadas à programação e em disciplinas de Cálculo Numérico que preveem a articulação com a programação. Outras proposições, como as encontradas nesta pesquisa, mas menos expressivas, extrapolam essas contribuições no desenvolvimento do TPACK. No entanto, é importante ressaltar, que, as contribuições no 
desenvolvimento do TPACK podem se dar para além do previsto na ementa das disciplinas, no sentido de que mesmo uma disciplina totalmente voltada para a programação tem o potencial de desenvolver conhecimentos pedagógicos, pedagógicos das tecnologias e os demais conhecimentos da estrutura TPACK. Esta afirmação é uma extrapolação das proposições de Lins (2003), quando afirma que, nas disciplinas matemáticas, está presente a formação pedagógica e, nas disciplinas pedagógicas, está presente a formação matemática, defendendo, portanto, a total impossibilidade de que ambos conhecimentos sejam trabalhados separadamente na formação do professor. Embora os conhecimentos que compõem o TPACK sejam de natureza diferente, na formação de professores é preciso que sejam estabelecidas e desenvolvidas de forma consistente as relações e ligações entre ambos. Esta posição se opõe a uma abordagem de que os conhecimentos do TPACK devam ser tratados separadamente na formação do professor, ou que um deva preceder outro.

Esse caminho para o desenvolvimento do TPACK se apresenta como uma opção bastante interessante para os cursos de Licenciatura, não só em Matemática, uma vez que os mesmos têm, diante de si, uma gama bastante grande e complexa de demandas formativas. No entanto, se torna crucial que essas possibilidades de desenvolvimento do TPACK por meio da programação (seja nas diferentes formas que se apresentam nos cursos de Licenciatura em Matemática) sejam esclarecidas e previstas no PP, na busca do rigor necessário ao currículo. (DOLL JR., 1997).

\section{REFERÊNCIAS}

ANDRÉ, M. E. D. A Estudo de caso em Pesquisa e Avaliação Educacional. Brasília: Líber Livro, 2005. BARDIN, L. Análise de conteúdo. Lisboa: Edições 70, 2007.

BRASIL. Ministério da Educação. Base Nacional Comum Curricular. 2017. Disponível em: http:// basenacionalcomum.mec.gov.br/. Acesso em: 10 jul. 2018.

BRASIL. Conselho Nacional de Educação. Parecer $n^{\circ}$ 2, de $1^{\circ}$ de julho de 2015. Diretrizes Curriculares Nacionais para a formação inicial em nível superior/Ministério da Educação. Diário Oficial da União, Brasília, 01 jul. 2015. p. 8-12.

BRASIL. Avaliação dos cursos de graduação: bacharelado e licenciatura, 2008. Disponível em: http:// www.inep.gov.br/download/superior/2009/Reconhecimento_Licen.pdf. Acesso em: 30 out. 2018.

BRASIL. Resolução CNE/CP 1, 18 de fevereiro de 2002. Diretrizes para a Formação Inicial de Professores da Educação Básica, em Cursos de Nível Superior. Diário Oficial da União, Brasília, 9 abr. 2002. Disponível em: http://portal.mec.gov.br/seesp/arquivos/pdf/res1_2.pdf. Acesso em: 30 out. 2018.

BRASIL. Parecer CNE/CES 1.302, 6 de novembro de 2001. Diretrizes Curriculares

Nacionais para os Cursos de Matemática, Bacharelado e Licenciatura. Diário Oficial da União, Brasília, 5 mar. 2002. Disponível em: http://portal.mec.gov.br/cne/arquivos/pdf/CES13022.pdf. Aceso em: 30 out. 2018.

COSTA, P. K. A. O blog na formação inicial de professores de matemática: oportunizando o contato com novas tecnologias. In.: RAUSKI, E. F.; BASSANI, F.; SANTOS, M. L. F. B.; RODRIGUES, M. F. (orgs.). Inovações educativas e ensino virtual: equipes capacitadas: práticas compartilhadas. Ponta Grossa: Estúdio Texto, 2016. p. 23-41. Disponível em: http://www.ufal.edu.br/arapiraca/graduacao/ matematica/projeto-pedagogico/ppc-matematica-2007/view. Acesso em: 10 jan. 2019.

DOLL JR., W. E. Currículo: uma perspectiva pós-moderna. Porto Alegre: Artes Médicas, 1997.

GAMA, C. L. G.; GOMES, M. N.; PIRES, L. A. Da teoria à prática: problematização e metodologias no Cálculo Numérico. Ensino em Re-Vista, Uberlândia, v. 25, n. 1, p. 234-255, jan./abr. 2018. Disponível em: http://www.seer.ufu.br/index.php/emrevista/article/view/41375/21883. Acesso em: 23 abr. 2019. 
GARLET, D.; BIGOLIN, N. M.; SILVEIRA, S. R. Uma proposta para o ensino de programação de computadores na educação básica. RESIGeT [online], Franca, v. 9, n. 2, p. 135-160, maio/ago. 2018. Disponível em: http://periodicos.unifacef.com.br/index.php/resiget/article/view/1604/1144. Acesso em: 23 abr. 2019.

IBGE. Instituto Brasileiro de Geografia e Estatística. Tabela 2387 - Domicílios particulares permanentes e Moradores em domicílios particulares permanentes por classes de rendimento mensal domiciliar e existência de microcomputador, acesso à Internet e tipo de telefone do período de 2014 a 2015. 2015. Disponível em: https://ww2.ibge.gov.br/home/estatistica/populacao/trabalhoerendimento/pnad2015/ sintese_defaultxls.shtm. Acesso em: 20 jun. 2018.

IF ES. Instituto Federal do Espírito Santo Campus Cachoeiro do Itapemirim. Projeto Pedagógico da Licenciatura em Matemática. Cachoeiro do Itapemirim, 2010. Disponível em: https://www.ci.ifes. edu.br/cursos/165-cursos/graduacao/12320-licenciatura-em-matematica-cachoeiro-de-itaprmirim. Acesso em: 23 abr. 2019.

IF FLUMINENSE. Projeto Pedagógico do curso de Licenciatura em Matemática do IF Fluminense Campus Centro, Campos de Goytacazes, 2012. Disponível em: http://licenciaturas.centro.iff.edu.br/ cursoslicenciatura/licenciatura-em-matematica/plano-pedagogico-do-curso/projeto-pedagogico-docurso-de-licenciatura-em-matematica/view?searchterm=PROJETO+PEDAG\%C3\%93GICO+MATEM \%C3\%81TICA. Acesso em: 30 nov. 2018.

IFBA. Instituto Federal da Bahia. Projeto Pedagógico do Curso de Licenciatura em Matemática Campus Valença. Valença, 2014. Disponível em: https://portal.ifba.edu.br/proen/departamentos/ departamento-de-ensino-superior/arquivo/ppc-superior-com-resolucao/valenca/licenciaturamatematica/projeto_curso_licenciatura_matematica_valenca_2014.pdf/@@download/file/Projeto_ Curso_Licenciatura_Matematica_Valen\%C3\%A7a_2014.pdf. Acesso em: 23 fev. 2019.

IFCE. Instituto Federal de Educação, Ciência e Tecnologia do Ceará. Projeto Pedagógico Licenciatura em Matemática Campus Cedro, Cedro, 2012. Disponível em:https://ifce.edu.br/cedro/campus_cedro/ cursos/superiores/licenciatura/matematica/pdf/projeto-pedagogico-licenciatura-em-matematicacampus-cedro.pdf/@@download/file/Projeto\%20Pedag\%C3\%B3gico\%20-\%20Licenciatura\%20 em\%20Matem\%C3\%A1tica\%20-\%20Campus\%20Cedro.pdf. Acesso em: 24 fev. 2019.

IFRS. Instituto Federal do Rio Grande do Sul. Projeto Pedagógico do Curso de Licenciatura em Matemática Campus Ibirubá. Ibirubá, 2014. Disponível em: https://ibiruba.ifrs.edu.br/site/midias/ arquivos/2014422153752141ppc_aprovado_2014.pdf. Acesso em: 28 jan. 2019.

IFTO. Instituto Federal do Tocantins. Projeto Pedagógico do Curso de Licenciatura em Matemática Campus Palmas. Palmas, 2015. Disponível em: http://www.ifto.edu.br/ifto/colegiados/consup/ documentos-aprovados/ppc/campus-palmas/licenciatura-em-matematica/ppc-licenciatura-matematicacampus-palmas-2edicao.pdf/view. Acesso em: 12 dez. 2018.

LINS, R. Os problemas da educação matemática. Folha OnLine, São Paulo, 29 abril 2003. Sinapse. Disponível em: http://www1.folha.uol.com.br/folha/sinapse/ult1063u385.shtml. Acesso em: 05 mar. 2019.

MASETTO, M.T. Competência pedagógica do professor universitário. São Paulo: Summus. 2012.

MENEGAIS, D. A. F. N.; FAGUNDES, L. C. F.; SAUER, L. Z. Impacto da Inserção de tecnologias digitais na formação inicial de professores de Matemática egressos de uma universidade pública federal. Novas Tecnologias na Educação, Porto Alegre, v. 12, n. 2, jul. 2014. Disponível em: https://seer.ufrgs. br/renote/article/view/53560. Acesso em: 30 out. 2018.

MOTTA, M. S. Formação inicial do professor de matemática no contexto das tecnologias digitais. Contexto \& Educação, Ijuí, v. 32, n. 102, p. 170-204, maio/ago. 2017.

ORO, N. et al. Olimpíada de programação de computadores para estudantes do ensino fundamental: a interdisciplinaridade por meio do Software Scratch. In.: WORKSHOP DE INFORMÁTICA NA ESCOLA, 21. 2015, Maceió. Anais [...] Porto Alegre: SBC, 2015. p. 102-111. 
PONTE, J. P.; CANAVARRO, P. Matemática e novas tecnologias. Lisboa: Universidade Aberta, 1997.

SBEM. Subsídios para a discussão de propostas para os cursos de Licenciatura em Matemática: uma contribuição da Sociedade Brasileira de Educação Matemática. 2003. Disponível em: http://sbembrasil. org.br. Acesso em: 20 jun. 2018.

SBM/SBEM. A formação do professor de Matemática no curso de Licenciatura: reflexões produzidas pela comissão paritária SBM/SBEM, 2013. Boletim SBEM, n. 21, fev. 2013. Disponível em: http:// sbembrasil.org.br/files/Boletim21.pdf. Acesso em: 20 jun. 2013.

SCAICO, P. D. et al. Ensino de programação no ensino médio: uma abordagem orientada ao design com a linguagem Scratch. Revista Brasileira de Informática na Educação, Porto Alegre, v. 21, n. 02, p. 92-103, maio/ago. 2013.

SOUZA, L. O. As TIC na formação docente: fundamentos para design de objetos virtuais de aprendizagem. 2016, 162 f. Dissertação (Mestrado em Educação em Ciências e Matemática) - Programa de Pós-Graduação em Educação em Ciências e Matemática, Universidade Federal de Goiás, Goiânia, 2016.

UDESC. Universidade do Estado de Santa Catarina. Projeto do Curso de Licenciatura em Matemática Campus Joinville. Joinville, 2015. Disponível em: http://www.joinville.udesc.br/portal/ensino/ graduacao/matematica/arquivos/PPC_Matematica.pdf. Acesso em: 2 jan. 2019.

UEMS. Universidade Estadual de Mato Grosso do Sul. Projeto Pedagógico do Curso de Licenciatura em Matemática Unidade Universitária de Cassilândia, Cassilândia, 2011. Disponível em: http:// www.uems.br/assets/uploads/cursos/cd531d21c9a8a51b06283670a82cef2c/projeto_pedagogico/1_ cd531d21c9a8a51b06283670a82cef2c_2015-02-09_11-57-20.pdf. Acesso em: 28 mar. 2019.

UFAL. Universidade Federal de Alagoas. Projeto Pedagógico do Curso de Licenciatura em Matemática Campus Arapiraca, Arapiraca, 2007. Disponível em: https://ufal.br/estudante/graduacao/ projetos-pedagogicos/campus-maceio/matematica-licenciatura-ead. Acesso em: 28 mar. 2019.

UFRN. Universidade Federal do Rio Grande do Norte. Projeto Pedagógico do Curso de Licenciatura em Matemática Campus Caicó. Caicó, 2010. Disponível em: https://sigaa.ufrn.br/sigaa/public/curso/ ppp.jsf?lc=pt_BR\&id=2000055. Acesso em: 20 mar. 2019.

UFSCAR. Universidade Federal de São Carlos. Projeto Pedagógico do Curso de Licenciatura em Matemática. São Carlos, 2005. Disponível: http://www.prograd.ufscar.br/cursos/cursos-oferecidos-1/ matematica/matematica-sao-carlos-licenciatura-projeto.pdf. Acesso: 20 fev. 2019.

UFV. Universidade Federal de Viçosa. Projeto Político Pedagógico Matemática-Licenciatura Integral e Noturno (Campus Viçosa). Viçosa, 2013. Disponível em: http://www.novoscursos.ufv.br/graduacao/ ufv/mtm/www/wp-content/uploads/2011/05/NOVO-PPC-MATEMATICA-Licenciatura-20-03-20133. pdf. Acesso em: 30 jan. 2019

UNESP. Universidade Estadual Paulista. Projeto Político Pedagógico com proposta de alteração do curso de Licenciatura em Matemática Campus Ilha Solteira. Ilha Solteira, 2014. Disponível em: https://www.feis.unesp.br/Home/Graduacao/cursos/projeto-politico-pedagogico.pdf. Acesso em: 21 fev. 2019.

UNICAMP. Universidade Estadual de Campinas. Projeto Pedagógico do Curso de Graduação em Matemática. Campinas, 2015. Disponível em: https://www.ime.unicamp.br/sites/default/files/ inline/349/ppp2015.pdf. Acesso em 24 mar. 2019.

VALENTE, J. A. Integração do pensamento computacional no currículo da educação básica: diferentes estratégias usadas e questões de formação de professores e avaliação do aluno. Revista e-Curriculum, São Paulo, v. 14, n. 3, p. 864-897, jul./set. 2016. Disponível em: https://revistas.pucsp. br/index.php/curriculum/article/view/29051/20655. Acesso em: 15 jul. 2018. 
VALENTE, J. A. O “estar junto virtual” como uma abordagem de educação a distância: sua gênese a aplicações na formação de educadores reflexivos. In.: VALENTE, J. A.; BUSTAMENTE, S. B. V. (orgs.) Educação a distância: prática e formação do profissional reflexivo. São Paulo: Avercamp, 2009. p. 37-64.

VALENTE, J. A. A espiral da aprendizagem e as tecnologias da informação e comunicação. In.: JOLY, M. C. R. A (org.). A tecnologia no ensino: implicações na aprendizagem. São Paulo: Casa do Psicólogo, 2002. p. 15-40.

VALENTE, J. A.; ALMEIDA, F. J. Visão analítica da informática na educação no Brasil: a questão da formação do professor. Revista Brasileira de Informática na Educação, Porto Alegre, v.1, n. 1, p. 45 60, jan./dez. 1997. Disponível em: https://www.br-ie.org/pub/index.php/rbie/article/view/2324/2083. Acesso: 30 set. 2018.

WING, J. Pensamento computacional: um conjunto de atitudes e habilidades que todos, não só cientistas da computação, ficaram ansiosos para aprender e usar. Revista Brasileira de Ensino de Ciências e Tecnologia, Ponta Grossa, v. 9, n. 2, p. 1-10, maio/ago. 2016. Disponível em: https://periodicos.utfpr. edu.br/rbect/article/view/4711/pdf. Acesso em: 15 jul. 2018. 